\title{
Hochschulbildung und Digitalisierung - Entwicklungslinien und Trends für die 2020er-Jahre
}

\author{
Markus Deimann
}

\section{Zusammenfassung}

Der Beitrag geht von der grundlegenden These aus, dass ein Blick in die Zukunft, ohne die Lehren der Vergangenheit zu berücksichtigen, ins Leere läuft. Dies trifft auch auf den hier verhandelten Gegenstand zu. So haben sich in einem Diskurs, der bis in die 1990er-Jahre zurückreicht, charakteristische Argumentationsmuster der Auseinandersetzung mit technologieunterstützter Hochschulbildung herausgebildet, die beständig aktualisiert werden. Auf diese geht der Beitrag ein, um damit eine kritische Auseinandersetzung mit dem Phänomen der Digitalisierung als ein Fortschrittsnarrativ vorzubereiten. Zum Abschluss wird vor dem Hintergrund der COVID-19-Pandemie eine andere Lesart von Digitalisierung, die enger an den Bedürfnissen der Hochschullehre ansetzt, eingeführt.

\section{Schlüsselwörter}

Hochschule • Hochschulbildung • Digitalisierung • E-Learning • Trends • COVID-19

\section{$1 \quad$ Einleitung}

Der vorliegende Beitrag hat eine andere Gestalt, als es ursprünglich geplant war. So sollte es schwerpunktmäßig um eine Beschreibung möglicher Szenarien für

M. Deimann ( $\varangle)$

Online-Landesportal ORCA.nrw, Bochum, Deutschland

E-Mail: markus.deimann@ ruhr-uni-bochum.de 
die Zukunft der digitalen Hochschulbildung gehen - eine gewaltige Herausforderung, wenn man sich die in den Medien dargestellte Dynamik technologischer Entwicklungen und die damit prognostizierten Auswirkungen auf Organisationen vor Augen führt. Wie aber lassen sich dann noch aussagekräftige Trends bestimmen, wenn unklar bleibt, wie sich Technologien auf gesellschaftliche Prozesse auswirken werden? Einerseits werden Innovationen ein großes disruptives Potenzial für die Bildung zugesprochen, andererseits zeichnen sich Hochschulen seit Jahrhunderten durch eine hohe Beständigkeit und Verlässlichkeit aus.

Hinzu kommt, dass die Zukunft insgesamt nicht mehr so attraktiv erscheint und die Faszination für Technologien als Motor für gesellschaftlichen Fortschritt ist der Befürchtung von damit verbundenen negativen sozialen und kulturellen Auswirkungen gewichen. Massenhafte Überwachung und Kontrolle, Fake News, Hatespeech und diskriminierende Algorithmen sind zu digitalen Phänomenen mit globaler Reichweite geworden.

Der Literaturwissenschaftler Hans Ulrich Gumbrecht beschreibt die merkwürdige Situation, in der wir uns seit einiger Zeit befinden, als „brüchige Gegenwart“ und meint damit:

„,[...] die Gegenwart dehnt sich immer weiter aus, so dass alles (und das Gegenteil von allem) in ihr möglich scheint, und der Zukunftshorizont verengt sich zur Erwartung einer kommenden - aber vielleicht noch fernen - Katastrophe" (Gumbrecht 2019, S. 107).

Aus dieser veränderten Konstellation von Vergangenheit, Gegenwart und Zukunft lassen sich folgende Schlussfolgerungen für die Gestaltung dieses Beitrags ziehen. $\mathrm{Zu}$ Beginn richte ich den Blick in die Vergangenheit und diskutiere unter der Perspektive „Das Erbe des E-Learnings“ das Zusammenspiel technischer und pädagogischer Entwicklungen im Zeitraum von Mitte der 1990er- bis Mitte der 2000er-Jahre und werfe einen Blick auf die darunterliegenden Mythen und Narrative, die bestimmte Vorstellungen vom Lernen mit digitalen Werkzeugen hervorgebracht haben. Diese tiefere Ebene wird häufig nicht explizit gemacht, sodass die prägenden Einflüsse der Narrative die Pädagogik überlagern und verzerren.

Mit dem Rückblick lässt sich, so meine These, die Wahrnehmung für Kontinuitäten und Transformationen der Digitalisierung von Hochschulbildung schärfen. Es geht nun um Plattformen, Daten und Künstliche Intelligenz, während weniger prestigeträchtige, dafür pädagogisch bedeutsame Themen wie Open Educational Resources und Open Educational Practices zurückgedrängt werden. 
Zum Abschluss gehe ich auf die COVID-19-Pandemie ein und die dadurch offensichtlich gewordene „digitale Kluft“, englisch Digital Divide, in Bezug auf die sozialen Imaginationen (Taylor 2004), die mit der Digitalisierung von Hochschulbildung verbunden sind. Damit gemeint sind die in der Gesellschaft verankerten Vorstellungen, was digitale Bildung ist und welche Zwecke sie zu erfüllen hat. Zwei soziale Imaginationen konkurrieren dabei um Aufmerksamkeit: 1) Digitalisierung von Bildung als generalstabmäßig zu bearbeitende Aufgabe und 2) eine romantisch verklärte Vorstellung von Hochschulbildung, die sich an der unhintergehbaren Bedeutung gemeinsamer Präsenz aufhängt. Die Bearbeitung dieser sich gegenüberstehenden Positionen wird den weiteren Verlauf der Debatte über die Zukunft der Hochschullehre in diesem Jahrzehnt bestimmen. Insofern erscheint eine Perspektive, die weniger deterministisch - in Bezug auf Technologien und das Soziale - argumentiert, als eine sinnvolle Alternative.

Noch ein kurzer Hinweis zum methodischen Vorgehen, das sich aus zwei Schritten zusammensetzt:

1. Für die Rekonstruktion des frühen E-Learning-Diskurses sowie der aktuellen Entwicklungen im Bereich Digitalisierung der Hochschule wird auf überblicksartige Darstellungen zurückgegriffen, die über gängige Datenbanken (zum Beispiel FIS Bildung) recherchierbar sind. Die Auswahl erfolgt anhand der Breite der Darstellungen, das heißt, es werden nur solche Beiträge in die Auswertung einbezogen, die aus einer Metaperspektive auf die E-LearningEntwicklung insgesamt blicken. Darstellungen einzelner Projekte sind daher von nachgelagertem Interesse.

2. Für die Identifizierung der darunter liegenden Imaginationen, Mythen und Narrative orientiere ich mich an Überlegungen aus der Technik- und Kulturphilosophie (zum Beispiel Mosco 2005) sowie der Organisationssoziologie (Clark 1983). Die dort beschriebenen, für die damalige Zeit typischen Denkmodelle (zum Beispiel die Kalifornische Ideologie) werden auf den E-Learning-Diskurs übertragen und anhand charakteristischer Aussagen illustriert.

\section{Das Erbe des E-Learning}

\subsection{Aufstieg und Fall der Neuen Medien}

Das Sprechen über Neue Medien wirkt im Jahr 2020 seltsam antiquiert. ELearning ist nun erwachsen geworden und entledigt sich seiner altmodischen 
Bezeichnungen, wie zum Beispiel Tele-Teaching, virtuelle Hochschule oder Multimedia Learning. Vor 20 Jahren war das anders, die neuen Medien hatten eine Scharnierfunktion und standen semantisch in Verwandtschaft zur Wirtschaft Neuer Markt - und der Politik - Neue Mitte. Dadurch wurde ein Gefühl von Modernisierung und des Aufbruchs in eine neue Zeit vermittelt. Auch die Bildung sollte modernisiert werden, dank der Segnungen der neuen Technologien. Wer wollte sich schon ernsthaft gegen den Fortschritt stellen? Diese suggestive Frage weist auf ein tiefergehendes Problem hin - die ökonomische Engführung von Modernisierung und die Anpassung von Hochschulbildung an die Erfordernisse des freien Marktes.

Genau diese ökonomisch geprägte Denkweise war ein zentraler Bestandteil eines in den 1990er-Jahren sehr einflussreichen Narrativs, des CyberLibertarismus. Ein wichtiger Aspekt betraf das Lebensgefühl der Gegenkultur, das sich in der Hippiebewegung rund um San Francisco manifestierte und die auf bizarre Weise mit einer Technikeuphorie zur Kalifornischen Ideologie verschmolzen wurde (Barbrook und Cameron 1995). Es sind eigentlich unvereinbare Positionen, die hier zusammenfanden: die linke Freiheitsbewegung und der Neoliberalismus. Vereint sind sie allerdings durch den Glauben an die Technik als die treibende Kraft zur Befreiung des Individuums von politischen und sozialen Beschränkungen sowie zur Verwirklichung eines neuen digitalen Markts. Dabei wurde das Internet als eine Cyberutopie konstruiert, die außerhalb aller politischen, ökonomischen und historischen Bezüge der Gesellschaft steht.

Die Faszination für den Neuen Markt wurde durch den Fall der New Economy Anfang der 2000er-Jahre nicht getrübt, sondern als eine Art vorübergehender Einbruch einer ansonsten unaufhaltsamen Revolution betrachtet. Die damalige Bundesministerin für Bildung und Forschung mahnte 2002 vor einer in Deutschland zu hoch ausgeprägten Internetresistenz (Bulmahn 2002), die dem Fortschritt im Weg stehe. Dem wollte das Bundesministerium für Bildung und Forschung mit der Förderinitiative „Neue Medien in der Bildung“ entgegenwirken, die damals mit einem Volumen von 185 Mio. EUR zu den finanzstärksten Initiativen weltweit gehörte (Kleimann und Wannemacher 2004).

Die Hoffnungen von E-Learning auf nachhaltige Qualitätsverbesserung und Kosteneinsparungen kamen genau zum richtigen Zeitpunkt, denn die Hochschulen befanden sich in keinem guten Zustand: knapper werdende Finanzmittel, steigende Studierendenzahlen sowie ein fortschreitender Wettbewerb mit kommerziellen Bildungsorganisationen. Es gab einen ausgeprägten Krisendiskurs mit Büchern wie „Im Kern verrottet. Fünf vor Zwölf an Deutschlands Universitäten“ (Glotz 1996) und gefragt waren neue Steuerungsmodelle, die für mehr Effizienz beim Management knapper Ressourcen sorgen sollten. 


\title{
2.2 Schlussfolgerungen - was vom E-Learning übrig bleibt
}

Mitte der 2000er-Jahre, also wenige Jahre nach dem von Politik und Wirtschaft ausgerufenen Aufbruch in ein neues digitales Zeitalter, sahen Seufert und Euler die Zukunft von E-Learning an einem Scheideweg angekommen:

,[...] entweder etabliert sich eLearning zunehmend als integraler Bestandteil der Lehre an Hochschulen, oder eLearning bleibt dort ein Fremdkörper und der bildungstechnologische Friedhof wird neben dem Schulfernsehen, der programmierten Instruktion und dem Sprachlabor um eLearning erweitert" (Euler und Seufert 2005, S. 2).

Die Einschätzung von Erfolg oder Scheitern von E-Learning hängt auf unhintergehbare Weise mit dem Standpunkt der Betrachtenden und der zugrunde liegenden politischen Agenda ab. Dadurch können Urteile unterschiedlich ausfallen und sich nur auf bestimmte Ausschnitte der E-Learning-Integration beziehen. Für den vorliegenden Rückblick betrifft das zum einen die faktische Ebene der E-Learning-Initiativen und Projekte sowie die Frage nach deren Nachhaltigkeit und zum anderen die Ebene der Narrative mit der Frage, welche E-Learning-Vorstellungen sich zugunsten anderer Ideen durchsetzen konnten.

Was auf der ersten Ebene erreicht wurde, lässt sich durch umfangreiche Dokumentationen und Berichte rekonstruieren. In der Übersicht „E-Learning-SupportEinrichtungen an deutschen Hochschulen“ (Kleimann und Wannemacher 2004) ist es ein günstiges Zusammenspiel von Faktoren wie Medienentwicklungsstrategie, Infrastruktur und Kooperationsbeteiligten. Ein weiteres Beispiel ist der „Sachstandsbericht zum Monitoring eLearning“, der zu einem konstruktiv-kritischen Resümee kommt:

\begin{abstract}
„Auch wenn die geplanten Ressourceneinsparungen, die hochschulischen Strukturveränderungen sowie die nachhaltigen Implementierungs- und Verstetigungskonzepte nicht in der gewünschten Form erreicht wurden, sind dennoch das Engagement zum Medieneinsatz an Hochschulen gefördert und enorme Potenziale freigelegt worden" (Revermann 2006, S. 197).
\end{abstract}

$\mathrm{Zu}$ einer ähnlichen Einschätzung, wonach E-Learning zwar in die Hochschule gekommen sei, aber noch nicht Alltag der Hochschullehre geworden ist, kommt die Studie „Adresse nicht gefunden - auf den digitalen Spuren der E-TeachingFörderprojekte“ (Haug und Wedekind 2009). Von Nachhaltigkeit könne angesichts fehlender Dokumentation kaum die Rede sein. Ebenfalls sei das große Ziel der Entwicklung von neuen Geschäftsmodellen für E-Learning an Hochschulen weitgehend nicht erreicht worden. 
Was bei dieser und ähnlichen Studien fehlt, ist eine tiefergehende Analyse, die über technische Infrastruktur und/oder organisatorische Rahmenbedingungen hinausgeht und auch die normativen Ideale und Wunschvorstellungen, die mit dem Einsatz von E-Learning an der Hochschule verknüpft sind, in den Blick nimmt. Eine solche Betrachtung offenbart, dass es zu unausweichlichen Konflikten im Sinne eines „Kampfes der Kulturen“ - angelehnt an den Begriff „Clash of Cultures“ von Samuel Huntington - kommen musste. Als ein prägnantes Beispiel lässt sich auf der einen Seite das marktwirtschaftlich orientierte Denken identifizieren sowie auf der anderen Seite die in einer Vielzahl von normativen Leitideen gefangenen Hochschulen. Darunter fallen Vorstellungen wie das klassische humboldtsche Bildungsideal, aber auch die Idee der Universität als „Fortschrittsagentur“ (Nassehi 2018). Im Unterschied zu Unternehmen der New Economy waren Hochschulen zumeist nicht profit-, sondern gemeinwohlorientiert und keinem Businessplan, sondern einem Curriculum verpflichtet. Diese Andersartigkeit wurde jedoch nicht als etwas, das genuin zur Hochschule gehört, begriffen, sondern eher als lästiger historischer Ballast, den man nun auf dem Weg in die Informationsgesellschaft rasch loswerden müsse.

Zurück zur Frage, was vom E-Learning übrigblieb. Die Bearbeitung der technischen, infrastrukturellen und organisatorischen Herausforderungen ging voran, wenn auch in einer für Hochschulen typischen Geschwindigkeit. Was dagegen weniger bearbeitet wurde, sind die Narrative, die mit E-Learning verknüpft waren. Die Herausforderung liegt nun darin, sie so zu explizieren und offenzulegen, dass sie mit den Idealen und der Wirklichkeit an der Hochschule kontrastiert werden können. Das betrifft insbesondere die von kommerziellen Anbieter*innen mit bestimmten Ideologien vermarkteten Bildungstechnologien, aber auch generell die von Politik und Wirtschaft verbreiteten Geschichten über die Zukunft von Hochschule in der digitalen Welt.

Insgesamt zeigt sich hier eine erstaunliche Stabilität und Anpassungsfähigkeit der Narrative. So wurden die empirisch oft nicht haltbaren Vorstellungen zur Flexibilität und Orts- und Zeitunabhängigkeit, die mit der Einführung von E-Learning stark gemacht wurden und zu weniger Kosten und mehr Lernleistung führen sollten, fortgeführt und übertragen auf die nachfolgenden Innovationen wie Blended Learning oder Mobile Learning - gleichwohl ohne dass sich in der Organisation von Hochschullehre strukturell etwas änderte. 


\section{$3 \quad$ Kontinuitäten und Transformationen: Vom E-Learning zur Digitalisierung von Hochschule}

Das Erbe des E-Learnings lässt sich als Normalisierung von Internettechnologien für den Einsatz in der Hochschullehre verstehen. Neue Medien haben ihren Neuigkeitseffekt verloren und sind, wenn auch auf divergierende Weise, Teil des Alltags geworden. Es scheint allerdings auch weiterhin wenig Konsens über eine E-Learning-Didaktik zu geben und die Entscheidung für eine bestimmte E-Learning-Variante ist oft mehr durch persönliche Präferenzen als durch didaktische Gründe beeinflusst. Es überwiegt nach wie vor ein instrumentelles Verständnis von Bildungstechnologien, das heißt der Glaube, damit irgendwie Lernen und Lehren zu verbessern. Wirkmächtig ist weiterhin das Denken in Kategorien analoger Hochschuldidaktik.

Auf diese kulturellen Kontinuitäten ist $\mathrm{zu}$ achten, wenn wir uns nun mit der Digitalisierung von Hochschulen als dem Megathema beschäftigen, das wie kaum ein anderes die 2020er-Jahre zu bestimmen scheint. Die sogenannte digitale Transformation ist in der populären Darstellung eine technologische Revolution (Schwab 2016) oder - übertragen auf die Hochschulbildung - eine „digitale Bildungsrevolution“ (Dräger und Müller-Eiselt 2015). Bei einer solch gewaltigen Rhetorik wirkt die zurückliegende Epoche des E-Learnings wie ein Sturm im Wasserglas. Denn nun geht es um die Hochschule insgesamt, um alle Funktionsbereiche und Leistungsaspekte.

Das typische Argumentationsmuster sieht meist wie folgt aus. Es gibt einen gewaltigen technologischen Wandel, wodurch es zu ,tektonischen Plattenverschiebungen in Gesellschaft und Organisation“ (Ehlers 2020, S. VIII) kommt. Daraus wird die Conclusio eines ,tiefgreifenden Wandels im Hochschulbereich“ abgeleitet, der nun dringend herbeigeführt werden müsse. Notwendig sind strategische Top-down-Entscheidungen, wie sie beispielsweise vom Hochschulforum Digitalisierung mit einer Peer-to-Peer-Beratung unterstützt werden (Röwert 2019). Wir sehen hier auch wieder den Einfluss eines technik-deterministischen Denkens, das Innovationen als unmittelbar wirkmächtig für die Gesellschaft versteht und der Hochschulbildung nur eine nachgelagerte Rolle zuweist.

Bei so viel Willen zur Veränderung stellt sich unweigerlich Skepsis gegenüber den vorgebrachten teleologischen Visionen ein. Auf einen Aspekt möchte ich exemplarisch eingehen, nämlich die Unvollständigkeit der Argumentation. Sie besagt, dass die Digitalisierung ein globales und alle gesellschaftlichen Bereiche betreffendes Phänomen sei, das quasi naturgesetzlich zu umfassenden Veränderungen führt. Am Beispiel der Wirtschaft (Industrie 4.0) oder der Medien (Netflix, Spotify) könne dieser Prozess schon studiert werden. Digitalisierung als 
universelles Phänomen wird dann dazu genutzt, um Hochschule in eine Reihe mit Wirtschafts- und Medienunternehmen zu stellen und Slogans wie „Bildung 4.0“ (Meinel 2017) abzuleiten. Diese dienen jedoch mehr der Positionierung von bestimmten Sprecher*innen in einem aufgeheizten Diskurs als der substanziellen Beschäftigung mit Bildungskonzepten. Dabei bleibt ausgeblendet, dass Hochschulen durchaus eigene Lösungsmechanismen entwickeln können. So gesehen wäre zunächst zu fragen, für welche Bildungsprobleme Digitalisierung die Lösung sein kann (siehe Nassehi 2019 vertiefend für eine solche Perspektive).

Bisher scheint allerdings der von außen an die Hochschule herangetragene Solutionismus (Morozov 2013) prägend zu sein, auch für die 2020er-Jahre. Das werde ich im nächsten Abschnitt mit den MOOCs als einem Teaser für die kommende digitale Zukunft, die sich um Personalisierung dreht, ermöglicht durch Algorithmen und Learning Analytics, aufzeigen. Ähnlich wie E-Learning gelten MOOCs heute nicht mehr als „hip“, zeigen aber anschaulich die Muster der Imagination von zukünftiger Hochschulbildung auf.

\subsection{Das digitale Erweckungserlebnis: MOOCs und Hochschule}

Kaum jemand konnte sich den Hype um Massive Open Online Courses vorstellen, der in der Proklamation des „Year of the MOOC“ (Pappano 2012) gipfelte, als vier Jahre zuvor kanadische Bildungsexpert*innen eine neue Form des vernetzten Lernens über das Internet präsentierten und dies als MOOC bezeichneten. Diese frühe Form, angelehnt an das Konzept des Connectivism (Siemens 2005) und der globalen Open-Education-Bewegung (Deimann 2018), war eine sehr anspruchsvolle und damit eigentlich für die Hochschulen passende Lernform. Vieles, was üblicherweise in Lehrveranstaltungen vorgegeben wird, wie die zu bearbeitende Literatur und die Themen der einzelnen Sitzungen, wurde von der Gemeinschaft der Lernenden selbst erarbeitet. Die Bezeichnung Dozierende wurde konsequenterweise durch "Course Facilitator" ersetzt. Auch in Deutschland gab es offene Kurse, die an das Konzept der konnektivistischen MOOCs angelegt waren (Bremer 2012). Diese wurden allerdings außerhalb eines kleinen Zirkels engagierter Menschen nicht wahrgenommen - ähnlich den ab 2001 aufkommenden offenen digitalen Bildungsressourcen (OER), die durch bestimmte Freiheitsrechte digital adäquates Lernen und Lehren erlaubten. OER stellen eine notwendige und hinreichende Bedingung für Bildung unter den Bedingungen der Digitalität dar, konnten sich aber bislang im Mainstreamdiskurs der Digitalisierung nicht nachhaltig etablieren. Das liegt, so meine Annahme, an der bis zum Ausbruch 
der COVID-19-Pandemie dominierenden Vorstellung von digitaler Bildung als Fortsetzung der analogen Hochschullehre mit digitalen Mitteln (zum Beispiel Learning-Management-System).

Ab 2011 kam der eigentliche MOOC-Hype, der sich weniger auf gemeinsames Netzlernen bezog, sondern auf die Vermittlung von standardisierten und videobasierten Inhalten an eine anonyme Masse auf einer geschlossenen Plattform. In der Öffentlichkeit wurde angesichts der radikalen Öffnung von Hochschule von der Revolutionierung der Bildung gesprochen (Dräger und Müller-Eiselt 2015). Über Sinn und Unsinn solcher und anderer Slogans ist mittlerweile viel geschrieben worden (siehe zum Beispiel Deimann 2015) und muss an dieser Stelle nicht nochmals wiederholt werden.

Von Bedeutung ist vielmehr, wie mit der populären xMOOC-Variante ${ }^{1}$ die Tür zu einem ersten breiten Diskurs über die Digitalisierung an Hochschulen geöffnet wurde. Dieser folgte in einem gewissen zeitlichen Abstand auf den E-LearningDiskurs und so ging es bei MOOCs auch immer um die sprichwörtliche Frage, ob alter Wein in neue Schläuche abgefüllt werde. Damit würde man MOOCs allerdings nicht gerecht, denn diese waren in einem viel stärkeren Maße in die Kultur der Hochschule integriert, als das beim E-Learning der Fall war. Dafür sorgte unter anderem der sogenannte MOOC-Production-Fellowship-Wettbewerb, der 2013 zusammen vom Stifterverband für die Deutsche Wissenschaft und dem Start-up Iversity durchgeführt wurde. Daraus entstand eine solche Sogwirkung, dass auch Hochschulen, die nicht prämiert wurden, in die Produktion von MOOCs investierten und so der Plattform von Iversity und dem Thema zusätzliche Aufmerksamkeit verschafften. Weitere hochschulpolitische Akteur*innen beteiligten sich an der Diskussion und brachten, wie etwa die Hochschulrektorenkonferenz, ihre Position ein. Darin wurde eine „Auseinandersetzung mit den Möglichkeiten einer Weiterentwicklung der digitalen Lehrformate" (Hochschulrektorenkonferenz 2014) ausdrücklich befürwortet. Während in dieser Empfehlung eine breite Perspektive auf digitale Lehre anklingt, wurde die Debatte tatsächlich stark auf MOOCs verkürzt.

Dieses Geschäftsfeld war wie bereits in der früheren E-Learning-Phase hauptsächlich von den USA und Großbritannien (später auch China) dominiert. ${ }^{2}$

\footnotetext{
${ }^{1}$ xMOOC steht für extended / erweitert, wobei ein traditioneller Hochschulkurs als Vorlesung oder Seminar über das Internet für alle Interessierten zugänglich gemacht wird. An der Tiefenstruktur der Hochschullehre ändert sich dadurch nichts, so dass die xMOOCs aus pädagogischer Sicht als wenig innovativ zu bewerten sind.

${ }^{2}$ Siehe dazu die seit 2015 jährlich herausgegebenen MOOC-Reports unter: https://www.cla sscentral.com/report. Zugegriffen: 06.07.2020.
} 
Deutsche Hochschulen verfügten meist nicht über die finanziellen und infrastrukturellen Mittel, um eigene MOOCs anzubieten, und waren dadurch auf Kooperationen mit privaten Anbietern - in Deutschland Iversity, in den USA Coursera, Udacity und MITx/edX - angewiesen.

Es zeigte sich dann rasch, dass die Situation in Deutschland sehr unterschiedlich zu den USA ist. So hatte beispielsweise Iversity nach der ersten Welle von fertig produzierten und ausfinanzierten MOOCs Schwierigkeiten, an Aktualisierungen oder neue Inhalte zu kommen, was schließlich in der Insolvenz mündete (Wirtschaftswoche 2016). Mittlerweile hat sich die Intensität und Vehemenz der Debatte um MOOCs gelegt. Dazu trug insbesondere die erste Phase des Hochschulforums Digitalisierung (HFD) von 2014 bis 2016 bei, das sich intensiv mit der Frage auseinandersetzte, ob die Hochschulen in naher Zukunft angesichts digitaler Disruption verschwinden würden. Das HFD ist ein Beispiel für die Diskursivierung der Digitalisierung von Hochschulbildung (siehe dazu Bedenlier und Deimann 2020), die von der intensiven Auseinandersetzung mit MOOCs ihren Ausgang nahm. Diese Zeit zwischen Hype und Beginn einer Normalisierung lässt sich damit als Phase der Weichenstellungen für den weiteren Kursverlauf betrachten.

\subsection{Der Aufstieg der Bildungsplattformen}

Im Unterschied $\mathrm{zu}$ den früheren MOOC-webbasierten Lernformaten und den Open Educational Resources, die das Internet als offenen digitalen Kulturraum begreifen, steht bei xMOOC die Plattform im Zentrum. Sie bietet als digitales Portal Zugang zu einer Vielzahl von hochwertigen Kursangeboten, zum Teil mit der Möglichkeit, dafür anrechenbare Creditpoints zu bekommen, die deshalb attraktiv für Studierende weltweit sind. Zudem lassen sich mit den digitalen Zertifikaten in Form von Badges neu erworbene oder vertiefte Kompetenzen feingranularer demonstrieren als mit klassischen Abschlusszeugnissen. So lautet, grob skizziert, die Begründung, warum im Zuge des MOOC-Hypes eine Reihe von kommerziellen Plattformen in den USA entstanden. Coursera, Udacity und edX begannen damit, die von Hochschulen produzierten Kurse unter ihrer Dachmarke zu vertreiben, und schufen so einen Bildungsmarkt (Ed-Tech), der in der frühen ELearning-Phase bereits imaginiert wurde, sich aber in Deutschland bis heute nicht etablieren konnte. Stattdessen greifen das Silicon-Valley-Prinzip und das darunterliegende Narrativ, mit dem zahllose Start-ups arbeiten (siehe dazu auch Liu 2020): Risikokapital wird von Investor*innen in mehreren Runden eingesammelt, das Unternehmen verspricht neue, innovative und effiziente Lösungen, wächst rasch 
und sucht nach Möglichkeiten, Profit zu generieren. Für MOOCs war das eine besondere Herausforderung, da sie per se kostenlos sein sollten. Das betraf aber nur die Inhalte und nicht die Zertifikate oder besondere Zusatzleistungen (zum Beispiel Recruiting).

Aus pädagogischer Sicht verfolgten die xMOOCs durch den Einsatz von Lehrvideos eine konservative Ausrichtung und übertrugen das dominante vorlesungsbasierte Modell von der Hochschule auf die digitale Plattform. Es war die Fortführung des aus der Fernlehre bekannten Broadcast-Modells, bei dem Inhalte von einer Institution an eine Vielzahl von Lernenden weltweit versendet werden. Einen Rückkanal gab es in Form von Korrespondenz oder Telefon, jedoch mit wenigen Möglichkeiten zur Interaktion untereinander. Kritisiert wurde auch eine Tendenz zum Bildungsimperialismus, da viele US-MOOCs auf Inhalte und Beispiele setzen, denen ein westliches Denk- und Wertemodell zugrunde lag (Schulmeister 2013). Die Vorherrschaft nordamerikanischer Elitehochschulen konnte so weiter ausgebaut werden, auch wenn MOOCs aus dem asiatischen Raum an regionalem Einfluss gewannen.

Durch die kommerzielle Vermarktung von Bildungsinhalten stehen die Plattformen im Widerspruch zur Philosophie der Open-Education-Bewegung, wonach Wissen ein freies, kollektives Gut ist (Deimann 2018). Auch wurde die Forderung nach Demokratisierung von Bildung konterkariert, da xMOOCs durch ihr besonderes didaktisches Design hauptsächlich Menschen mit hoher akademischer Vorbildung anzogen, während die Masse an sozial schlechter gestellten Lernenden nicht erreicht wurde (Knox 2019) - genau das wurde jedoch mit dem Ausspruch der Revolutionierung versprochen.

Während also pädagogisch-didaktische Aspekte eher eine marginale Rolle bei der Entwicklung und Etablierung einer Reihe von einflussreichen MOOCPlattformen spielten, waren ökonomisch-strategische Faktoren umso bedeutsamer. Entsprechend dem prägenden Silicon-Valley-Narrativ des Cyber-Libertarismus (siehe Abschn. 2.1) waren es widersprüchliche Versprechungen, mit denen der Aufstieg dieser und zukünftiger Bildungsplattformen vollzogen wurde: auf der einen Seite das Versprechen der Befreiung von sozialen und kulturellen Beschränkungen durch Technologien, auf der anderen Seite die zunehmende Abhängigkeit von der Plattform durch Lock-in-Effekte und dem Ausgesetztsein verschiedener Methoden der ,digitalen Ausbeutung“. Damit wurde der Weg in eine datengetriebene Bildung (Data-driven Education) vorgeprägt. 


\subsection{Datafizierung}

Die Digitalisierung schreitet in den 2020-Jahren weiter voran, teilweise auf den Pfaden, die zuvor in der frühen E-Learning-Phase wie auch im späteren MOOC-Hype ausgetreten wurden. Darüber hinaus erleben wir jedoch mit der Datafizierung eine völlig neue Entwicklung, mit weitreichenden Auswirkungen auf die Organisation und das Verständnis von Hochschullehre (Hartong 2020; Pangrazio und Selwyn 2020). Mit Datafizierung sind dabei im Wesentlichen drei Aspekte verbunden: 1) Generierung/Produktion von digitalen Daten, 2) Auswertung und Verarbeitung digitaler Daten und 3) Verwendung der Daten mit sozialen, wirtschaftlichen und politischen Konsequenzen. Daten können sowohl aktiv, zum Beispiel über die Teilnahme an einem Onlinekurs auf einer Plattform oder passiv, etwa als GPS-Tracking, produziert, gesammelt und ausgewertet werden. Insgesamt hat die Verbreitung von algorithmen-basierten Dateninfrastrukturen so zugenommen, dass der Einsatz in nahezu allen gesellschaftlichen Bereichen alltäglich geworden ist (Cheney-Lippold 2017). Der Bildungsbereich ist von der Datafizierung besonders betroffen:

,[...] because it transforms not only the ways in which teaching and learning are organised but also the ways in which future generations (will) construct reality with and through data" (Jarke und Breiter 2019, S. 1).

Wichtig ist darum - der grundlegenden These des Beitrags folgend -, die Narrative, sozialen Imaginationen und ideologischen Aufladungen in den Blick zu nehmen, die mit digitalen Technologien und Datafizierung konstruiert werden. So wird argumentiert, dass sich Lernprozesse mit mehr Daten optimieren lassen, zum Beispiel sozial gerechter oder inklusiver, oder dass digitale Technologien völlig neue Formen der Personalisierung bieten, die der herkömmlichen pädagogischen Praxis weit überlegen sind. Datenbasierte Bildungsentscheidungen werden so als präzise, rational und wirklichkeitsgetreu dargestellt.

Tatsächlich sind sie das aber keineswegs. Entgegen der vielfach verbreiteten Vorstellung, dass Daten lediglich die Realität abbilden, lässt sich mit Verweis auf die Critical Data Studies (Macgilchrist 2018) ein Verständnis entgegenhalten, wonach Daten niemals neutral sind, sondern in ihnen immer schon bestimmte Wertvorstellungen eingeschrieben sind. Welche das sind - wie beispielsweise im Zusammenhang mit Learning Analytics -, ist eine dringend zu diskutierende grundlegende Fragestellung. So hat die Learning-Analytics-Forschung in den vergangenen Jahren eine augenscheinlich so überzeugende empirische Basis aufgebaut, dass es nur selbstverständlich erscheint, die suggestive Frage zu stellen: 
„Are Higher Education Institutions Prepared for Learning Analytics?“ (Ifenthaler 2017).

Versteht man Hochschulen als Einrichtungen, die sich einem bestimmten Bildungsverständnis verpflichtet haben, dann geht mit der Datafizierung eine fundamentale Transformation dieses Verständnisses einher. Es ist dann nämlich nicht mehr das Individuum mit seiner einzigartigen Persönlichkeit Adressat von Bildungsangeboten, sondern die von einer Person hinterlassenen Datenpunkte und -spuren, die maschinell verarbeitet und kategorisiert werden. Inwieweit dann noch von Bildung die Rede sein sollte, ist eine Frage, die ich an die Leser*innen weitergeben möchte.

Ungeachtet dieser Visionen datengetriebener Hochschullehre, die uns in den 2020er-Jahren weiter beschäftigen werden und für die sich die (Medien-)Didaktik und Bildungswissenschaft konzeptionell wappnen sollten, zeichnet sich aktuell noch eine andere Zukunft ab. Diese berührt unmittelbar nahezu alle mit der Hochschule beschäftigten Menschen.

\section{$4 \quad$ Ausblick: Digitalisierung als neue Normalität der Hochschullehre}

Es war ein in der jüngeren Geschichte der Hochschulen einmaliges Ereignis, als im März 2020 bedingt durch die COVID-19-Pandemie überall in Deutschland die Türen der Hörsäle geschlossen wurden. Die Präsenzlehre wurde zum „Notfallbetrieb“ erklärt und musste kurzfristig mit digitalen Werkzeugen und Inhalten sichergestellt werden (Schwartmann 2020) - eine gewaltige Herausforderung, ja Herkulesaufgabe, auf die sich allerdings laut einer Sonderbefragung des Hochschul-Barometers (2020) die allergrößte Mehrheit (knapp $90 \%$ ) der beteiligten Hochschulen gut vorbereitet sah. Tatsächlich waren die technischen und kulturellen Voraussetzungen für \#PivotOnline nicht so günstig und das wäre auch bei einem Blick zurück in die Geschichte des E-Learnings (Abschn. 2.2) deutlich geworden.

So steht zwar an nahezu allen Hochschulen eine IT-Infrastruktur zur Verfügung und es gibt in Rechenzentren und E-Learning-Serviceeinrichtungen vielfältige Beratungs- und Unterstützungsangebote für den Einsatz digitaler Medien in der Lehre. Dazu kommen standardmäßig Learning-Management-Systeme zum Einsatz und dienen der Ergänzung von Vorlesung und Seminar. Soll nun die Vorlesung oder das Seminar komplett online durchgeführt werden, reicht die dafür notwendige Ausstattung nicht aus, insbesondere im Bereich der Videokonferenzsysteme. Darum mussten kurzfristig Lizenzen kommerzieller Systeme wie etwa 
Zoom eingekauft werden, was zu kontroversen Diskussionen bezüglich Datenschutz und Datensicherheit führte. ${ }^{3}$ Auch verläuft der Einsatz der verschiedenen Bildungstechnologien oft unkoordiniert und ist durch die persönliche Motivation der Lehrenden sowie durch die Marketinganstrengungen der Softwarefirmen beeinflusst. Je nach pädagogischem Bedarf gibt es von anpassbaren LearningApps bis hin zu komplett vorproduzierten Inhalten auf Plattformen eine große Bandbreite an digitalen Dienstleistungen.

Ungeachtet dessen zeigt sich an der im Frühsommer 2020 aufgekommenen hitzigen Debatte um den Status der Präsenzlehre ${ }^{4}$, dass es die Hochschulen bislang nicht geschafft haben, ihre kulturellen Grundlagen an die Bedingungen des digitalen Zeitalters anzupassen. Es scheint weiterhin einen Kern unhinterfragter Vorstellungen und Annahmen darüber zu geben, was höhere Bildung ist, was ihr Sinn und Zweck ist und wie die Hochschulen diese organisieren. Dazu gehört etwa die Überzeugung, dass Bildung zwingend an regelmäßige Präsenz gekoppelt ist. Diese starre Formatierung der Lehre in Bezug auf Ort und Zeit ist eine zentrale Hürde. Auch existieren bislang keine einheitlichen und verbindlichen Weiterbildungsprogramme „Digitale Kompetenzen für die Lehre“, sondern die Qualifizierung ist dem persönlichen Engagement der Hochschullehrer*innen überlassen.

Das vielfach ungeklärte Verhältnis von Bildung und Bildungstechnologien betrifft jedoch nicht nur die Seite der Skeptiker*innen, sondern auch die Seite der Strateg*innen. Diese hoffen, dass COVID-19 zu einem Katalysatoreffekt und nachhaltigen Veränderungen im Lehrbetrieb führt und man dadurch die Phase der Pilotierungen von Einzelsystemen verlässt. Dazu wurde schon vor einigen Jahren mit der Formulierung von Digitalisierungsstrategien begonnen und zur Überprüfung der Umsetzung entstanden Stellen mit militärisch klingendem Titel (Chief Digital Officer). Die Frage stellt sich, ob diese Top-down-Ansätze nicht an der Realität der Lehrpraxis vorbeilaufen und sich Parallelwelten bilden ohne ausreichende Interaktion miteinander.

Es ist nun genau die Zeit, um mit dem Austausch und der Klärungsarbeit zu beginnen. Die nun vorhandenen Erfahrungen mit der „Quick-and-DirtyDigitalisierung" machen deutlich, dass es langfristiger Planungen und einer hohen Sensibilisierung für die Kraft der Digitalisierungsmythen bedarf. Ratsam sind darum kleine Schritte, die für die Mehrheit der Beteiligten machbar sind und

\footnotetext{
${ }^{3}$ Siehe dazu das Interview mit dem Landesbeauftragten für Datenschutz und Informationsfreiheit Baden-Württemberg: https://www.baden-wuerttemberg.datenschutz.de/datenschutzf reundliche-technische-moeglichkeiten-der-kommunikation/. Zugegriffen: 29.06.2020.

${ }^{4}$ Siehe: https://www.praesenzlehre.com/. Zugegriffen: 30.06.2020.
} 
die von einer gemeinsamen Vision von Hochschule in der Kultur der Digitalität ausgehen. Notwendig sind dabei vor allem organisatorische und dienstrechtliche Änderungen (zum Beispiel Lehrverordnung) zur Anpassung an die neuen Bedingungen. So gesehen könnte der Einsatz von E-Learning und digitalen Medien zu einer Selbstverständlichkeit werden und eine neue Normalität in der Hochschullehre begründen.

\section{Literatur}

Barbrook, R., \& Cameron, A. (1995). The Californian ideology. Mute, 1(3), 44-72.

Bedenlier, S., \& Deimann, M. (2020). „Bildung“ und „Digitalisierung“ im Spiegel von Digitalisierungsstrategien. Zeitschrift für Hochschulentwicklung, 15(1), 41-59.

Bremer, C. (2012). Open Online Courses als Kursformat? Konzept und Ergebnisse des Kurses „Zukunft des Lernens“ 2011. In G. Csanyi, F. Reichl, \& A. Steiner (Hrsg.), Digitale Medien. Werkzeuge für exzellente Forschung und Lehre. Medien in der Wissenschaft, 61 (S. 153-164). Münster: Waxmann.

Bulmahn, E. (2002). New Economy - Herausforderungen für die Bildungs- und Forschungspolitik. In G. Alt, H. Bill, \& M. Machnig (Hrsg.), Innovation. Technik. Zukunft (S. 39-50). Wiesbaden: VS Verlag.

Cheney-, J. (2017). We are data: Algorithms and the making of our digital selves. New York: New York University Press.

Clark, B. R. (1983). The higher education system: Academic organization in cross-national perspective. Berkeley: University of California Press.

Deimann, M. (2015). Die erfundene Revolution. HFD-Blog. https://www.hochschulforumd igitalisierung.de/blog/administrator/markus-deinmann-erfundene-revolution-digitale-bil dungsrevolution. Zugegriffen: 26. Okt. 2020.

Deimann, M. (2018). Open Education. Auf dem Weg zu einer offenen Hochschulbildung. Bielefeld: Transcript.

Dräger, J., \& Müller-, R. (2015). Die digitale Bildungsrevolution: Der radikale Wandel des Lernens und wie wir ihn gestalten können (1. Aufl.). München: DVA.

Ehlers, U.-D. (2020). Future Skills. Lernen der Zukunft-Hochschule der Zukunft. Wiesbaden: Springer VS.

Euler, D., \& Seufert, S. (2005). Von der Pionierphase zur nachhaltigen Implementierung - Facetten und Zusammenhänge einer pädagogischen Innovation. In D. Euler \& S. Seufert (Hrsg.), E-Learning in Hochschulen und Bildungszentren (S. 1-24). München: Oldenbourg.

Glotz, P. (1996). Im Kern verrottet? Fünf vor zwölf an Deutschlands Universitäten. Stuttgart: Deutsche Verlags-Anstalt.

Gumbrecht, H. U. (2019). Brüchige Gegenwart. Reflexionen und Reaktionen. Stuttgart: Reclam.

Hartong, S. (2020). Zum Optimierungsdrang des Bildungsmonitorings. Zeitschrift für Pädagogik, 66(1), 64-71. 
Haug, S., \& Wedekind, J. (2009). „Adresse nicht gefunden“ - Auf den digitalen Spuren der E-Teaching-Förderprojekte. In U. Dittler, J. Krameitsch, N. Nistor, C. Schwarz, \& A. Thilosen (Hrsg.), E-Learning: Eine Zwischenbilanz. Kritischer Rückblick als Basis eines Aufbruchs (S. 19-37). Münster: Waxmann.

Hochschul-Barometer. (2020). Große Mehrheit der Hochschulen sieht sich gut gerüstet für den Semesterstart. Hochschul-Barometer. https://www.hochschul-barometer.de/aktuell. Zugegriffen: 29. Okt. 2020.

Hochschulrektorenkonferenz. (2014). HRK-Positionspapier zu MOOCs im Kontext der digitalen Lehre. HRK. https://www.hrk.de/positionen/beschluss/detail/hrk-positionspapierzu-moocs-im-kontext-der-digitalen-lehre/. Zugegriffen: 6. Juli 2020.

Ifenthaler, D. (2017). Are higher education institutions prepared for learning analytics? TechTrends, 61(4), 366-371.

Jarke, J., \& Breiter, A. (2019). Editorial: The datafication of education. Learning, Media and Technology, 44(1), 1-6.

Kleimann, B., \& Wannemacher, K. (2004). E-Learning an deutschen Hochschulen. Von der Projektentwicklung zur nachhaltigen Implementierung (Hochschulplanung, 165). Hannover: HIS.

Knox, J. (2019). What does the "Postdigital" mean for education? Three critical perspectives on the digital, with implications for educational research and practice. Postdigital Science and Education, 1, 357-370.

Liu, W. (2020). Abolish Silicon Valley: How to liberate technology from capitalism. London: Repeater.

Macgilchrist, F. (2018). The "Digital Subjects" of twenty-first century education: On datafication, educational technology and subject formation. In S. Jagger \& P. Pericles (Hrsg.), Routledge Handbook of Cultural Studies in Education (S. 239-254). New York: Routledge.

Meinel, C. (2017). Bildung 4.0 - Wie wir morgen lernen werden. Heinrich-Böll-Stiftung. https://www.boell.de/de/2017/06/19/bildung-40-wie-wir-morgen-lernen-werden. Zugegriffen: 26. Okt. 2020.

Morozov, E. (2013). To save everything, click here: The folly of technological solutionism. New York: PublicAffairs.

Mosco, V. (2005). The digital sublime: Myth, power, and cyberspace. Cambridge: MIT Press.

Nassehi, A. (2018). Wozu Universitäten? Eine Legitimationsgeschichte. In A. Nassehi \& P. Felixberger (Hrsg.), Kursbuch 193-301 Gramm Bildung (S. 115-133). Hamburg: Kursbuch Kulturstiftung gGmbH.

Nassehi, A. (2019). Muster. Theorie der digitalen Gesellschaft. München: DTV.

Pangrazio, L., \& Selwyn, N. (2020). Towards a school-based „Critical Data Education“. Pedagogy, Culture \& Society, Advanced Online Publication. https://doi.org/10.1080/146 81366.2020.1747527.

Pappano, L. (2012). The year of the MOOC. The New York Times, (2. November). https:// www.nytimes.com/2012/11/04/education/edlife/massive-open-online-courses-are-mul tiplying-at-a-rapid-pace.html. Zugegriffen: 16. Nov. 2020.

Revermann, C. (2006). eLearning in Forschung, Lehre und Weiterbildung in Deutschland. Sachstandsbericht zum Monitoring eLearning (Arbeitsbericht, 107). Berlin: Büro für Technikfolgen-Abschätzung beim Deutschen Bundestag. 
Röwert, R. (2019). Unterstützung von Strategien für Hochschulbildung im digitalen Zeitalter durch Peer-to-Peer-Beratungen. In S. Robra-Bissantz, O. J. Bott, N. Kleinefeld, K. Neu, \& K. Zickwolf (Hrsg.), Teaching trends 2018 (S. 43-50). Münster: Waxmann.

Schulmeister, R. (2013). Der Beginn und das Ende von OPEN. Chronologie der MOOCEntwicklung. In R. Schulmeister (Hrsg.) MOOCs-Massive Open Online Courses. Offene Bildung oder Geschäftsmodell? (S. 17-62). Münster: Waxmann.

Schwab, K. (2016). Die vierte industrielle Revolution. München: Pantheon Verlag.

Schwartmann, R. (2020). Online-Lehre: Ein Pandemieplan für Hochschulen. FAZ.NET. https://www.faz.net/1.6734167. Zugegriffen: 29. Okt. 2020.

Siemens, G. (2005). Connectivism: A learning theory for the digital age. International Journal of Instructional Technology and Distance Learning, 2(1), 3-10.

Taylor, C. (2004). Modern social imaginaries. Durham: Duke University Press.

Wirtschaftswoche. (2016). Geplatzte Finanzierung: E-Learning-Start-up Iversity verkündet Insolvenz. Wirtschaftswoche, (19. Juli). https://gruender.wiwo.de/iversity-das-e-learningstartup-musste-insolvenz-anmelden/. Zugegriffen: 16. Nov. 2020.

Open Access Dieses Kapitel wird unter der Creative Commons Namensnennung 4.0 International Lizenz (http://creativecommons.org/licenses/by/4.0/deed.de) veröffentlicht, welche die Nutzung, Vervielfältigung, Bearbeitung, Verbreitung und Wiedergabe in jeglichem Medium und Format erlaubt, sofern Sie den/die ursprünglichen Autor(en) und die Quelle ordnungsgemäß nennen, einen Link zur Creative Commons Lizenz beifügen und angeben, ob Änderungen vorgenommen wurden.

Die in diesem Kapitel enthaltenen Bilder und sonstiges Drittmaterial unterliegen ebenfalls der genannten Creative Commons Lizenz, sofern sich aus der Abbildungslegende nichts anderes ergibt. Sofern das betreffende Material nicht unter der genannten Creative Commons Lizenz steht und die betreffende Handlung nicht nach gesetzlichen Vorschriften erlaubt ist, ist für die oben aufgeführten Weiterverwendungen des Materials die Einwilligung des jeweiligen Rechteinhabers einzuholen.

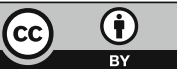

